\title{
Multivariate empirical mode decomposition and application to multichannel filtering
}

\author{
Julien Fleureau $^{1,2}$, Amar Kachenoura ${ }^{1,2}$, Laurent Albera ${ }^{1,2}$, \\ Jean-Claude Nunes ${ }^{1,2}$, Lotfi Senhadji ${ }^{1,2}$ \\ ${ }^{1}$ Inserm, UMR 642, Rennes, F-35000, France \\ ${ }^{2}$ Université de Rennes 1, LTSI, Rennes, F-35000, France \\ For correspondence: Amar Kachenoura \\ Tel : (33)-2232345 52, E-Mail : amar.kachenoura@univ-rennes1.fr
}

\begin{abstract}
Empirical Mode Decomposition (EMD) is an emerging topic in signal processing research, applied in various practical fields due in particular to its data-driven filter bank properties. In this paper, a novel EMD approach called X-EMD (eXtended-EMD) is proposed, which allows for a straightforward decomposition of mono- and multivariate signals without any change in the core of the algorithm. Qualitative results illustrate the good behavior of the proposed algorithm whatever the signal dimension is. Moreover, a comparative study of X-EMD with classical mono- and multivariate methods is presented and shows its competitiveness. Besides, we show that X-EMD extends the filter bank properties enjoyed by monovariate EMD to the case of multivariate EMD. Finally, a practical application on multi-channel sleep recording is presented.
\end{abstract}

Keywords: Mono- and multivariate empirical mode decomposition, filter bank structure, electroencephalography data analysis. 


\section{Introduction}

The Empirical Mode Decomposition (EMD) is raising great interest since its first appearance in the nineteens [1]. Indeed, it received wide attention in various fields such as biomedical engineering [2, 3], space research [4], hydrology [5], synthetic aperture radar [6], speech enhancement [7], watermarking [8], etc. EMD aims at decomposing sequentially a given signal into the sum of Amplitude and Frequency Modulated (AM/FM) zero-mean oscillatory signals, called Intrinsic Mode Functions (IMFs), plus a non-zero mean low-degree polynomial remainder. Each IMF is computed by using an iterative procedure, named sifting process, applied to the residual multicomponent signal. One sifting iteration consists: i) in computing the local mean of the residual signal, and ii) in subtracting it from the residue. This process is repeated until convergence to a designated IMF. Generally, the local mean is calculated as the half sum of the upper and the lower envelopes, obtained by interpolating between local minima and maxima, respectively, using cubic splines [1].

Since the original work on EMD [1], several studies have been presented. Some ones aim at giving a theoretical framework to monovariate EMD $[9,10$, 11] in order to make easier its analysis. Other ones underline the behavior of original EMD [1] on simulated data such as its data-driven filter bank structure in the case of broadband noise [12, 13]. Eventually, EMD procedure devoted to bivariate [14, 15, 16], trivariate [17] and, recently, multivariate signals $[18,19,20]$ have been proposed. The first bivariate approach in [14] uses the real parts of analytic and anti-analytic components of the bivariate signal which leads to two sets of complex-valued IMFs corresponding to the 
positive and negative frequency components of the signal, respectively. The second bivariate technique presented in [15] defines the extrema of a complex signal as points where the angle of the first derivative of the signal vanishes. In [16], two bivariate algorithms based on more geometrical considerations are developed. These two last approaches compute a local mean by averaging the monodimensional EMD's of projections of the original bivariate signal onto different angular planes. Rehman et al. proposed a trivariate approach [17] which is based on the computation of the extrema by projecting the input signal in multiple directions in $3 \mathrm{D}$ spaces via a quaternion rotation framework. The multivariate extension of EMD, developed in [18], can be seen as a generalization of the concept employed in the Rilling's bivariate [16] and the Rehman's trivariate [17] EMD. The mean envelope is obtained by averaging multiple envelopes, generated by taking projections of the multivariate signal along multiple directions on hyperspheres. Nevertheless, none of these techniques is a straightforward extension of the original monovariate EMD concept [1] to multivariate signals, say signals defined from $\mathbb{R}$ to $\mathbb{R}^{D}$ with $D>1$. It is probably due to the difficulty of interpreting the notion of extremum when multivariate signals are considered.

In recent works, [19, 20], we proposed an EMD algorithm, called 2T-EMD (Turning Tangent EMD), ables to process mono- and multivariate signals whatever the signal dimension $D$ is, without any change in the algorithm. In addition, 2T-EMD algorithm seems to require a smaller amount of calculations than classical EMD algorithms. To do so, the signal mean trend is redefined as the signal obtained by interpolating the barycenters of particular oscillations, called elementary oscillations (see section II [19, 20]). More pre- 
cisely, 2T-EMD algorithm is based on two main steps: i) identification of all elementary oscillations and ii) computation of the barycenters of each associated elementary oscillations and interpolation between all these barycenters to obtain the signal mean trend. Recall that, in [19, 20], an elementary oscillation of a given function $s$ with values in $\mathbb{R}^{D}(D \geq 1)$ is considered as a piece of $s$ defined between two consecutive oscillation extrema of $s$.

In this paper, we propose to compute the local mean trend without calculation of the oscillation barycenters (as proposed in 2T-EMD), giving rise to a new multivariate EMD algorithm, named X-EMD (eXtended EMD). Indeed, in X-EMD algorithm (as described in section 2), the local mean is directly computed by averaging two envelopes generated directly from oscillation extrema. In other words, the exploitation of oscillation extrema is clearly different between X-EMD and 2T-EMD algorithms and makes X-EMD algorithm easier both to implement and to use. The numerical complexity of the proposed approach is then analyzed in section 3 and compared with the complexity of four existing EMD methods. A performance study is proposed in section 4 on synthetic mono-, bi-, and trivariate signals showing: i) the competitive behavior of X-EMD versus existing methods, and ii) its ability to process any output signal dimension. Finally, data-driven spectrum analyzer properties satisfied by X-EMD are highlighted and a biomedical application is considered in section 5. Indeed, empirical filter bank structure enjoyed by monovariate EMD [13] is shown to be preserved by X-EMD in multivariate context. Such results is used to analyze a quadrivariate ElectroEncephaloGraphic (EEG) sleep recording. 


\section{An eXtended EMD approach: X-EMD}

The proposed X-EMD method aims at providing a unique tool able to process mono- and multivariate signals without any modification. The notion of oscillation extrema, originally presented in [19, 20], is exploited to redefine the local mean operator. However, before going further, let's briefly recall the definition of oscillation extrema.

Oscillation extremum. In [1], the local mean is given by taking average of the upper and the lower envelopes, which are computed by interpolating between the local maxima and minima, respectively. However, such points cannot be used for higher output signal dimensions, since they are not defined directly in multivariate contexts. To deal with this problem, we propose $[19,20]$ a new and general way to compute extrema in multidimensional output space $\mathbb{R}^{D}$.

Let $s$ be a function defined from $\mathbb{R}$ to $\mathbb{R}^{D}$ and differentiable with a continuous first derivative, say in class $\mathcal{C}^{1}$. Let define the function tangent vector to $s$ by:

$$
\boldsymbol{T}_{s}: t \longmapsto\left[1, \frac{d s}{d t}(t)\right]^{\top}
$$

Based on the following definition:

$$
\alpha_{s}: t \longmapsto \lim _{h \rightarrow 0}\left\langle\boldsymbol{T}_{s}(t-h), \boldsymbol{T}_{s}(t+h)\right\rangle
$$

where $\langle\cdot, \cdot\rangle$ denotes the Euclidean inner product of $\mathbb{R}^{D+1}$, the function $\alpha_{s}$ allows us to identify direction changes in function $s$. Due to the continuity of the inner product, it is noteworthy that function $\alpha_{s}$ can be rewritten as:

$$
\forall t \in \mathbb{R}, \quad \alpha_{s}(t)=\left\langle\lim _{h \rightarrow 0} \boldsymbol{T}_{s}(t-h), \lim _{h \rightarrow 0} \boldsymbol{T}_{s}(t+h)\right\rangle
$$


Now, since the considered function $s$ is in $\mathcal{C}^{1}, \alpha_{s}$ takes the following form:

$$
\forall t \in \mathbb{R}, \quad \alpha_{s}(t)=\left\|\boldsymbol{T}_{s}(t)\right\|^{2}=1+\left\|\frac{d s}{d t}(t)\right\|^{2}
$$

where $\|\cdot\|$ abusively denotes the Euclidean norm of both $\mathbb{R}^{D+1}$ and $\mathbb{R}^{D}$. So, oscillation extrema may be defined as the local minima of the following $\beta_{s}$ function:

$$
\beta_{s}: t \longmapsto \beta_{s}(t)=\left\|\frac{d s}{d t}(t)\right\|^{2}
$$

For monovariate signals, the reader can check that the oscillation extrema, computed from function $\beta_{s}(5)$, include the classical scalar extrema used in Huang's solution [1] but also the saddle points of $s$. It is noteworthy that oscillation extrema are different of inflection points [9] which require to compute the second order derivative of $s$ and which are not defined in multivariate contexts.

Local mean and sifting process. The key point of the X-EMD algorithm is the redefinition of the local mean, which allows us to use all oscillation extrema of the considered signal whatever its output dimension is. More particularly, the local mean is computed as the mean of two envelopes: a first envelope interpolating the even oscillation extrema (with signal borders) and a second envelope interpolating the odd oscillation extrema (also with signals borders). One could check that, for monovariate signals without any saddle points, this approach is equivalent to the original EMD method [1]. As far as the sifting process is concerned, we need to specify how to stop it. In fact, we use a classical modified Cauchy-like criterion. More precisely, let $d_{n, k}$ be the $n$-th IMF computed at the $k$-th iteration of the 
sifting process. Then, the sifting process is stopped when for instance $90 \%$ of values $\left\|d_{n, k+1}(t)-d_{n, k}(t)|||| d_{n, k}(t)\right\|$ are lower than $10^{-2}$.

\section{Numerical complexity analysis}

This section aims at relating the X-EMD algorithm and some existing EMD methods from a computational complexity point of view. Numerical complexity is calculated in terms of number of floating point operations (flops). A flop corresponds to a multiplication followed by an addition. But in practice, only multiplications are counted since, in general, there are about as many (and slightly more) multiplications as additions. The first considered EMD method is Huang's solution [1] and will be named Huang in the sequel. The second studied standard EMD approach is the second geometrical bivariate solution proposed in [16] and called Rilling hereafter. As far as the cases of multivariate signals are concerned, two methods, namely 2T-EMD algorithm [19, 20] and Rehman's method [18], are considered.

For a given EMD algorithm, let $N, K_{n}$, and $L$ be the number of extracted IMFs, the number of sifting iterations performed to extract the $n$-th IMF, and the data length, respectively. Now, denote by $d_{n, k}$ the $n$-th IMF computed at the $k$-th iteration of the sifting process, by: i) $M_{\mathrm{H}}\left(d_{n, k}\right)$ the number of extrema detected in $d_{n, k}$ by Huang [1], ii) $M_{\mathrm{R}}\left(d_{n, k}, p\right)$ the number of extrema detected in the $p$-th projection of $d_{n, k}$ using Rilling [16] when $P$ projection planes are used, iii) $M_{\mathrm{RM}}\left(d_{n, k}, p\right)$ the number of extrema detected in the $p$-th projection of $d_{n, k}$ by Rehman [18] when $P$ projection directions are used, iv) $M_{2 \mathrm{~T}}\left(d_{n, k}\right)$ the number of oscillation barycenters detected in $d_{n, k}$ by 2T-EMD [19, 20], and $\mathrm{V}) M_{\mathrm{X}}\left(d_{n, k}\right)$ the number of oscillation extrema detected in $d_{n, k}$ by X- 


\begin{tabular}{lll}
\hline Method & $D$ & Numerical complexity $F\left(d_{n, k+1}\right)$ \\
\hline Huang [1] & 1 & $18 L+15 M_{\mathrm{H}}\left(d_{n, k}\right)$ \\
Rilling [16] & 2 & $L(11 P+2)+15 \sum_{p=1}^{P / 2} M_{\mathrm{R}}\left(d_{n, k}, p\right)$ \\
Rehman [18] & $\mathbb{N}^{*}$ & $L P(2 D+18)+15 \sum_{p=1}^{P} M_{\mathrm{RM}}\left(d_{n, k}, p\right)$ \\
2T-EMD [19, 20] & $\mathbb{N}^{*}$ & $D\left(19 L+16 M_{2 \mathrm{~T}}\left(d_{n, k}\right)\right)+M_{2 \mathrm{~T}}\left(d_{n, k}\right)$ \\
X-EMD & $\mathbb{N}^{*}$ & $D\left(19 L+15 M_{\mathrm{X}}\left(d_{n, k}\right)\right)$ \\
\hline
\end{tabular}

Table 1: Computational complexity for one sifting iteration of X-EMD and four existing EMD methods. L and D represent the data length and the data dimension, respectively. Where $M_{\mathrm{H}}\left(d_{n, k}\right), M_{\mathrm{R}}\left(d_{n, k}, p\right), M_{\mathrm{RM}}\left(d_{n, k}, p\right), M_{2 \mathrm{~T}}\left(d_{n, k}\right)$ and $M_{\mathrm{X}}\left(d_{n, k}\right)$ denote the number of extrema detected in $d_{n, k}$ by Huang, the number of extrema detected in the $p$-th projection of $d_{n, k}$ by Rilling (when $P$ projection planes are used), the number of extrema detected in the $p$-th projection of $d_{n, k}$ by Rehman (when $P$ projection directions are used), the number of barycenters detected in $d_{n, k}$ by 2T-EMD, and the number of oscillation extrema detected in $d_{n, k}$ by X-EMD, respectively.

EMD. For given values of $L$ and $D$, the computational complexity, $F\left(d_{n, k+1}\right)$, of one sifting iteration necessary to obtain $d_{n, k+1}$ from $d_{n, k}$ is given in table 1 for Huang, Rilling, Rehman, 2T-EMD and X-EMD methods. Note that these results were obtained for a standard tridiagonal implementation of the spline interpolation and a signal from $\mathbb{R}$ to $\mathbb{R}^{D}$. Finally, the total computational cost of the five methods is obtained by summing the elementary complexities given in table 1 over the number of iterations, $K_{n}$, and the number of IMFs, $N$, as shown below:

$$
C(s)=\sum_{n=1}^{N} \sum_{k=1}^{K_{n}} F\left(d_{n, k}\right)
$$




\section{Performance evaluation of X-EMD}

The objective of this section is to compare the performance of X-EMD algorithm with classical approaches and to illustrate its ability to process multivariate signals whatever their output dimension. Before applying XEMD on test signals, it is prudent to give some details about the set of signals that X-EMD can decompose successfully. As previously mentioned, the considered signal has to be in class $\mathcal{C}^{1}$ or at least, in the case of irregular signals, an appropriate numerical estimation of the derivative has to be proposed. For instance, the first order derivative may be computed by means of a centered finite difference when forward and backward finite differences have the same signs and set to 0 otherwise. In addition, if $\left\{d_{n}\right\}_{1 \leq n \leq N}$ represents the theoretical set of IMFs composing the signal to analyze, it is obvious that any $d_{n}$ with a piecewise constant function $\beta_{d_{n}}(5)$ is not visible by X-EMD (a piecewise constant function has no local minimum). It concerns, for instance, monovariate signals with piecewise linear IMFs, and bivariate signals with purely circular rotating IMFs.

Performance criteria. Two criteria are used to evaluate the X-EMD algorithm and compare it to the existing EMD methods. The first criterion allows us to evaluate the efficiency of EMD algorithms to accurately extract one or all expected IMFs. Given $I$ a subinterval of $T=[-1,2]$, let's define the following quadratic errors:

$$
e_{I}\left(d_{n}\right)=\frac{\int_{I}\left\|d_{n}(t)-\hat{d}_{n}(t)\right\|^{2} d t}{\int_{I}\left\|d_{n}(t)\right\|^{2} d t} \quad \text { and } \quad e_{I}(s)=\sum_{n=1}^{N} e_{I}\left(d_{n}\right)
$$

where $\hat{d}_{n}$ denotes the estimate of the exact $n$-th IMF $d_{n}$ of signal $s$. More precisely, if we consider the interval, $I=[0,1]$, where border effects should 
be low, equation (7) allows us to compute a performance independent of any border effects. On the contrary, if $I=[-1,0] \cup[1,2]$ and for the same border management of all algorithms, equation (7) allows us to evaluate the ability of a given algorithm to minimize border effects. Indeed, such effects are often critical, especially, in real life data and their minimization should facilitate the practical exploitation of the IMFs. The second criterion, $C(s)$, evaluates the numerical complexity of each EMD algorithms, with respect to the formulas listed in table 1 .

Signal selection. To evaluate the behavior of X-EMD algorithm, two monovariate $\left(s_{11}, s_{12}\right)$ signals of the form $s_{1 i}=\sum_{n} d_{n}^{(1 i)}$, two bivariate signals $\left(s_{21}\right.$, $\left.s_{22}\right)$ of the form $s_{2 i}=\sum_{n} d_{n}^{(2 i)}$ and a trivariate $\left(s_{31}\right)$ signal of the form $s_{3 i}=\sum_{n} d_{n}^{(3 i)}$, defined on the time interval $T=[-1 ; 2]$ and sampled at $f_{s}=1 \mathrm{kHz}$, are tested. The mono- and bivariate signals are given by (succes- 
sive IMFs are in curly brackets):

$$
\begin{aligned}
s_{11}(t)= & d_{1}^{(11)}(t)+d_{2}^{(11)}(t) \\
= & \left.\left\{0.5 e^{0.23(1+t)} \sin (20 \pi t)\right\}+\left\{e^{0.23(1+t)} \sin (10 \pi t)\right)\right\} \\
s_{12}(t)= & d_{1}^{(12)}(t)+d_{2}^{(12)}(t)+d_{3}^{(12)}(t)+\text { Residue } \\
= & \{2 \sin (50 \pi t)\}+\left\{e^{0.23(1+t)} \cos \left(\pi(1.41 t+9.90)^{2}\right)\right\} \\
& +\left\{3 \cos \left(\pi(t+6)^{2}\right)\right\}+3 t \\
s_{21}(t)= & d_{1}^{(21)}(t)+d_{2}^{(21)}(t) \\
= & \left\{e^{\frac{i \pi}{4}} e^{t} \sin (80 \pi t+1.5)\right\}+\left\{e^{\frac{-\mathrm{i} \pi}{4}} \sin \left(2 \pi[2.5(1+t)]^{2}\right)\right\} \\
s_{22}(t)= & d_{1}^{(22)}(t)+d_{2}^{(22)}(t)+d_{3}^{(22)}(t) \\
= & \{0.3 \cos (56 \pi t)+0.8 \mathrm{i} \sin (54 \pi \mathrm{t})\}+\{1.7 \cos (20 \pi \mathrm{t})+3.2 \mathrm{i} \sin (22 \pi \mathrm{t})\} \\
& +\left\{e^{0.23 t-0.46}\left[2.5 \cos \left(\pi(0.82 t+4.49)^{2}\right)+1.1 \mathrm{i} \sin \left(\pi(0.82 \mathrm{t}+4.49)^{2}\right)\right]\right\}
\end{aligned}
$$

and the trivariate signal is defined by $s_{31}=d_{1}^{(31)}+d_{2}^{(31)}+d_{3}^{(31)}$ with:

$$
\begin{aligned}
d_{1}^{(31)}(t)= & {\left[2.5 \sin (100 \pi t), 2 \cos (100 \pi t), e^{0.11 t+0.70} \sin (100 \pi t)\right]^{\top} } \\
d_{2}^{(31)}(t)= & {\left[5 e^{0.14(1+t)} \cos \left(\pi(t+13)^{2}\right), 2 e^{0.14(1+t)}\right.} \\
& \left.\sin \left(\pi(t+13)^{2}\right), 0.5 \sin (28 \pi t+1.2)\right]^{\top} \\
d_{3}^{(31)}(t)= & {[4 \sin (12 \pi t), \cos (12 \pi t), 6 \sin (14 \pi t+1.5)]^{\top} }
\end{aligned}
$$

From a general point of view, the proposed synthetic signals are the sum of multiple generic AM-FM components. More precisely, the monovariate signal $s_{11}$ is the sum of two exponential AM components containing saddle points. The signal $s_{12}$ is a more general AM-FM signal with a linear residue. Regarding the bivariate signals, $s_{21}$ is composed of two orthogonal AM-FM planar components whereas $s_{22}$ is the sum of different AM-FM elliptic rotat- 
ing components. Finally the trivariate signal $s_{31}$ is the sum of three general AM-FM components.

Qualitative multivariate decompositions. This section is dedicated to qualitatively illustrate the behavior of X-EMD in various multivariate contexts. Thus, the decompositions of the monovariate signal $s_{12}$, the bivariate signal $s_{21}$, and the trivariate signal $s_{31}$ computed on the time interval $[0 ; 1]$ are respectively depicted in figures 1, 2 and 3. Figure 1 presents the exact (dashed line) and estimated (plain line) IMFs. The correct linear shape of the residue illustrates the good behavior of the X-EMD algorithm in a monovariate case. Regarding the bivariate signal $s_{21}$ depicted in figure 2 , the two rotated planar IMFs are well extracted, with a low residue. As far as the trivariate signal is concerned, the figure 3 (a) represents the original signal (dark line) and the associated local mean (gray line) on the restricted $[0.5 ; 0.6]$ time interval and the figure 3 (b) displays the exact (dashed line) and the estimated (plain line) IMFs of the signal projected on the three main axis, namely X, Y and Z. The local mean seems to nicely go through the original signal, and the comparison between the estimated and exact projections of the IMFs shows the ability of the X-EMD method to perform trivariate decomposition without any change in the core of the algorithm.

Comparative study. This section adopts a more quantitative point of view and is dedicated to the comparison of X-EMD performance with those obtained by Huang's algorithm [1] in monovariate case, Rilling's method [16] in a bivariate context, Rehman's approach [18] for trivariate signals, and 2T-EMD [19, 20] methods for all tested signals. More precisely, the second 

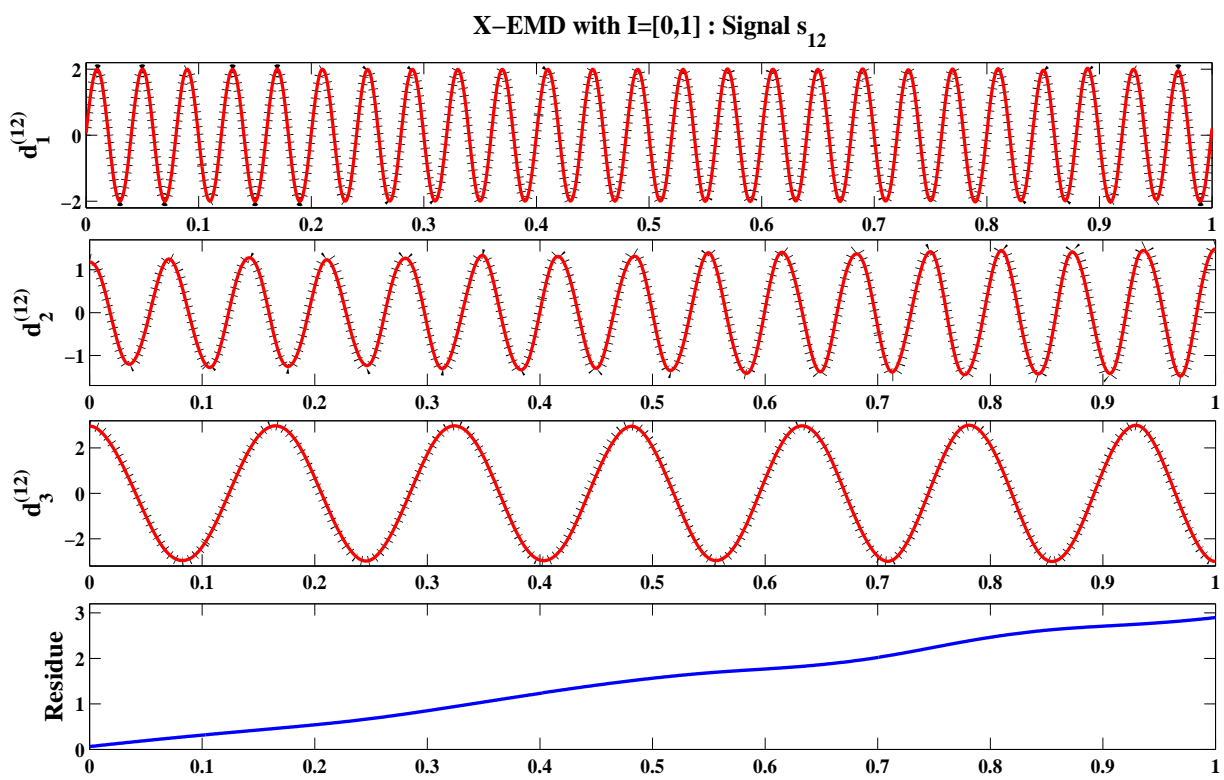

Figure 1: X-EMD monovariate decomposition of the signal $s_{12}$. Dashed line: real IMF / plain line: estimated IMF. 


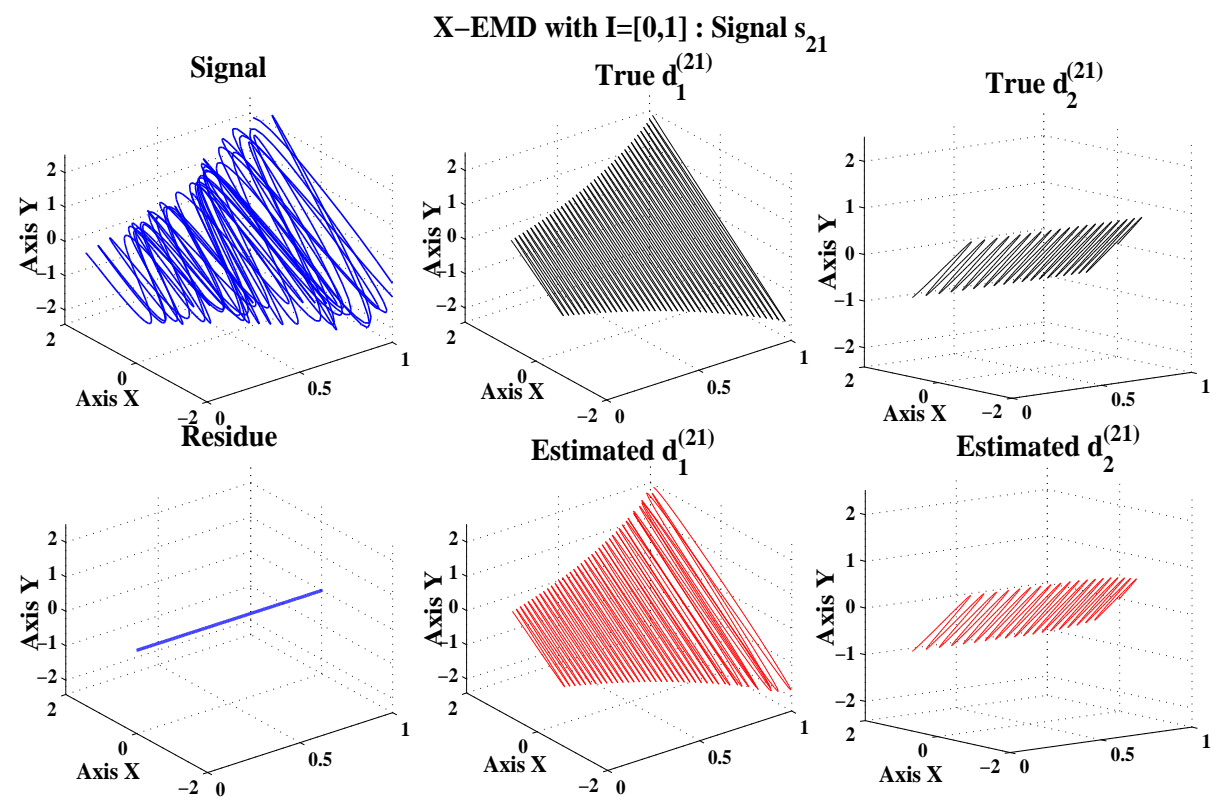

Figure 2: X-EMD bivariate decomposition of the signal $s_{21}$.

geometric approach proposed in [16] is used as reference for bivariate decompositions with $P=8$ projection planes. For Rehman's method we take $P=2 D$ projection directions on the associated hypersphere. Note that, the sifting process termination criterion and the border effect management are identical for all compared methods. This comparison is achieved in terms of performance using criteria $e_{[0,1]}(s)$ and $e_{[-1,0] \cup[1,2]}(s)$, but also in terms of numerical complexity using criterion $C(s)$. Results are provided in table 2 . It appears that for no border effects $(I=[0,1])$, the monovariate signal $s_{11}$ with saddle points is better decomposed by our approach in comparison to the Huang's method. In the bivariate case, Rilling's algorithm seems to be slightly more efficient on the rotating signal $s_{22}$ but X-EMD seems to offer a better performance on the signal $s_{21}$ made of rotated planar components. 
(a) $\mathrm{X}$-EMD zoom in $\mathrm{I}=[\mathbf{0 . 5 , 0 . 6}]$ : Signal $\mathrm{s}_{31}$

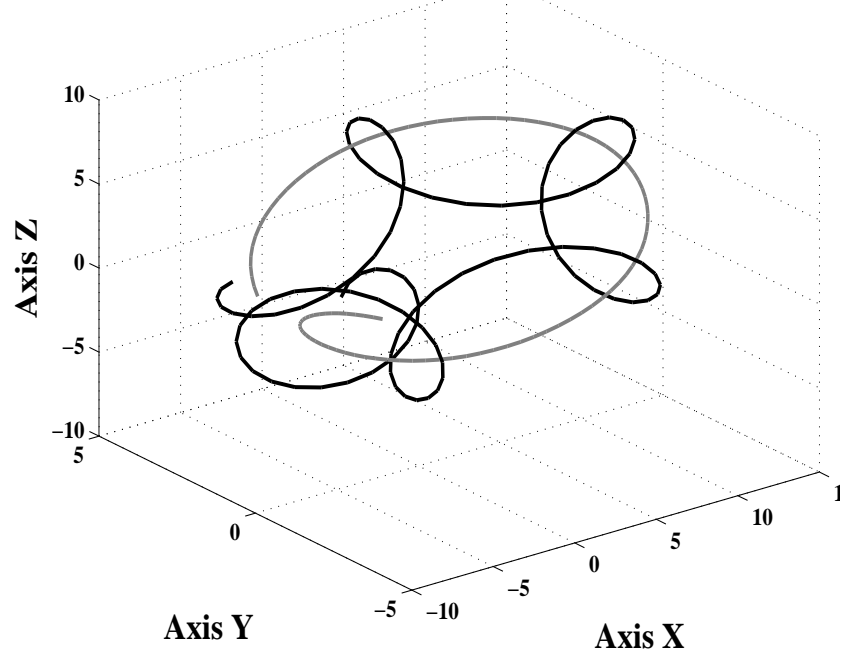

(b) Estimated IMF's projected on the three main axis : Signal s

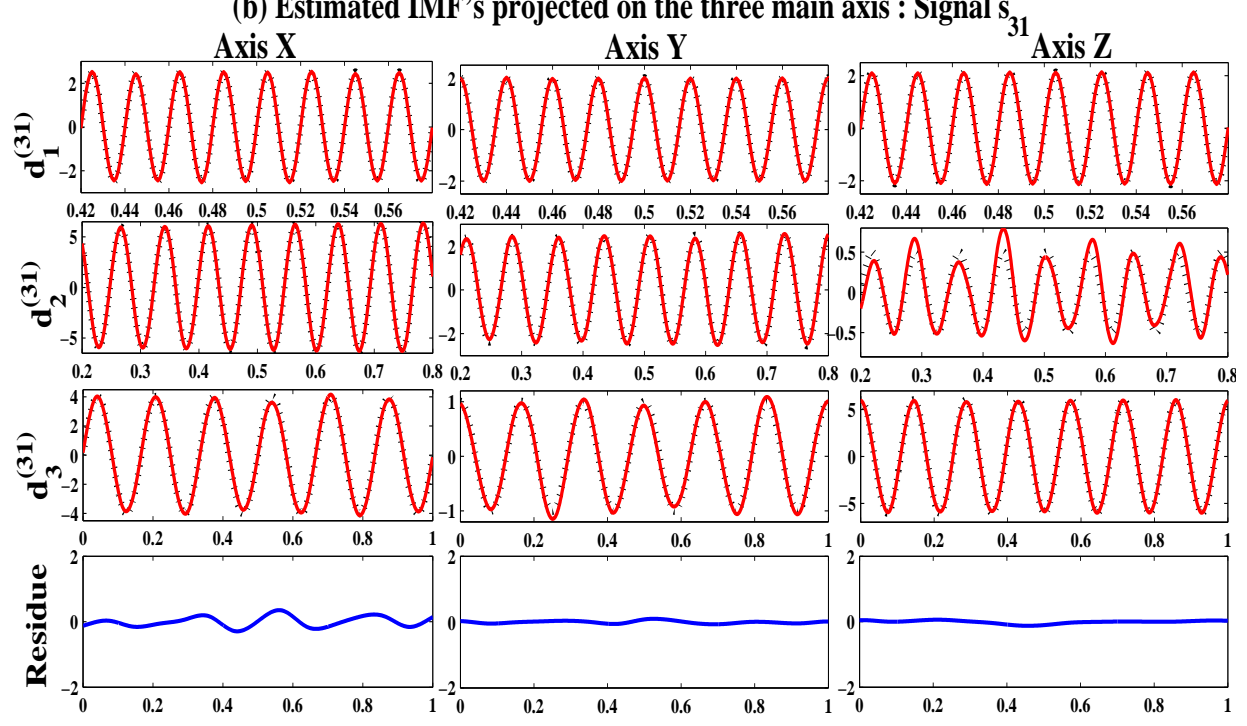

Figure 3: X-EMD trivariate decomposition of the signal $s_{31}$ on $[0 ; 1]$ : (a) original signal (dark) and its X-EMD local mean (gray), and (b) exact (dashed line) and estimated (plain line) IMFs projected on the three main axis $\mathrm{X}, \mathrm{Y}$ and $\mathrm{Z}$. 
Regarding the trivariate signal $s_{31}$, X-EMD method leads to better result than Rehman's algorithm. As far as the border effects are concerned, XEMD seems to have quasi-identical performance than Huang's method, and provides a more accurate management of border effects in comparison to the Rilling's and Rehman's methods. Concerning the computational complexity, table 2 clearly shows that for $D=1$ the computational complexities of X-EMD and Huang's method are quasi-equivalent. On the contrary, for $D=2$ and $D=3, \mathrm{X}$-EMD requires less sifting iterations and less computational operations than Rilling's and Rehman's methods. When focusing on the comparison between X-EMD method and 2T-EMD method (say the two methods which exploit, in different manner, the oscillation extrema), table 2 exhibits that the behavior of the two methods, using criteria $e_{[0,1]}(s)$ and $e_{[-1,0] \cup[1,2]}(s)$, are globally equivalent. More finely, X-EMD seems to provide a more accurate management of border effect for AM-FM signal $s_{12}$, where as $2 \mathrm{~T}$-EMD gives better border effects management in case of signal $s_{11}$ with saddle points. However, in accordance with the formulas presented in table 1 , the proposed X-EMD generally requires less computational operations than 2T-EMD method.

\section{Filter bank structure and application to multichannel sleep record- ing}

One important property of the classical monovariate EMD [1] especially highlighted in [12] is its empirical filter bank structure observed when EMD is applied on broadband noises. 


\begin{tabular}{|c|c|c|c|c|c|c|c|c|c|}
\cline { 2 - 11 } \multicolumn{1}{c|}{} & \multicolumn{3}{|c}{$e_{I}(s)$ with $I=[\mathbf{0 , 1}]$} & \multicolumn{2}{c|}{$e_{I}(s)$ with $I=[-1,0] \cup[1,2]$} & \multicolumn{3}{c|}{$C(s)$} \\
\hline Monovriate & Huang & 2T-EMD & X-EMD & Huang & 2T-EMD & X-EMD & Huang & 2T-EMD & X-EMD \\
\hline$s_{11}$ & $5.15 \mathrm{e}-3$ & $2.40 \mathrm{e}-3$ & $1.25 \mathrm{e}-3$ & $2.07 \mathrm{e}-1$ & $0.17 \mathrm{e}-1$ & $2.00 \mathrm{e}-1$ & $3.22 \mathrm{e}+6$ & $1.45 \mathrm{e}+6$ & $3.11 \mathrm{e}+6$ \\
\hline$s_{12}$ & $2.89 \mathrm{e}-3$ & $1.71 \mathrm{e}-3$ & $2.97 \mathrm{e}-3$ & $9.76 \mathrm{e}-2$ & $1.36 \mathrm{e}-1$ & $9.71 \mathrm{e}-2$ & $3.90 \mathrm{e}+6$ & $4.85 \mathrm{e}+6$ & $3.88 \mathrm{e}+6$ \\
\hline Bivariate & Rilling & 2 T-EMD & X-EMD & Rilling & 2T-EMD & X-EMD & Rilling & $2 \mathrm{~T}-$ EMD & X-EMD \\
\hline$s_{21}$ & $13.6 \mathrm{e}-3$ & $4.20 \mathrm{e}-3$ & $4.46 \mathrm{e}-3$ & $30.5 \mathrm{e}-1$ & $4.76 \mathrm{e}-1$ & $5.52 \mathrm{e}-1$ & $2.01 \mathrm{e}+7$ & $1.56 \mathrm{e}+7$ & $0.46 \mathrm{e}+7$ \\
\hline$s_{22}$ & $2.50 \mathrm{e}-3$ & $4.35 \mathrm{e}-3$ & $3.94 . \mathrm{e}-3$ & $5.80 \mathrm{e}-1$ & $4.25 \mathrm{e}-2$ & $4.21 \mathrm{e}-2$ & $1.95 \mathrm{e}+7$ & $0.72 \mathrm{e}+7$ & $0.60 \mathrm{e}+7$ \\
\hline Trivariate & Rehman & $2 \mathrm{~T}-$-EMD & X-EMD & Rehman & 2T-EMD & X-EMD & Rehman & $2 \mathrm{~T}-$ EMD & X-EMD \\
\hline$s_{31}$ & $5.65 \mathrm{e}-3$ & $1.19 \mathrm{e}-3$ & $0.91 \mathrm{e}-3$ & $1.07 \mathrm{e}-1$ & $0.42 \mathrm{e}-1$ & $0.46 \mathrm{e}-1$ & $3.58 \mathrm{e}+7$ & $0.59 \mathrm{e}+7$ & $0.22 \mathrm{e}+7$ \\
\hline
\end{tabular}

Table 2: Comparative study of X-EMD versus Huang (1D), Rilling (2D), Rehman (2D) and 2T-EMD (1D, 2D and 3D) reference methods.

Filter bank structure. The goal of this section is to show how this property is preserved by X-EMD for higher output signal dimensions. Nevertheless, the notion of frequential content for multivariate signals may be ambiguous. As suggested in [13], one alternative way to deal with IMFs frequency in a monovariate context is to consider their number of zero-crossings or alternatively their number of extrema, $N_{\text {ext }}$ (as those quantities differ at most by one in the definition of an IMF). In the same sense, we propose here to evaluate the frequential content of a mono- or multivariate IMF by considering its number of oscillation extrema. It is worth to point out that all the results presented hereafter do not take into account the first mode, $d_{n, k}$ $(\mathrm{k}=1)$, because as shown in $[12,13]$ the filter associated to the first mode is essentially highpass. In a first simulation, 500 uncorrelated mono-, bi- and trivariate white noises (1024 samples, with normal distribution) are generated and decomposed using X-EMD. For each IMF $d_{n, k}(\mathrm{k}>1)$ and for each 
signal, $N_{\text {ext }}$ is computed and a linear regression model is then fitted on the $\log _{2}$ averaged number of oscillation extrema $\left(\log _{2}\left(\overline{N_{e x t}}\right)\right)$ as depicted in figure 4 . The linear regression quality shows how $N_{\text {ext }}$ exponentially decreases with the number of IMFs, and suggests a filter bank structure whatever the considered dimension is. This bank appears close to a dyadic filter bank for a monovariate decomposition ( -0.65 slope in $\log _{2}$ coordinates) and the exponential decrease seems to be lower with the dimensionality increase $(-0.17$ slope for the hexavariate $D=6$ decomposition). This result strongly suggests that a higher number of IMFs could be expected for decompositions with higher dimensionality.

In a second simulation, the influence of spatial correlation between signal channels on the filter bank structure is studied. To do so, the previous simulation is repeated in a bivariate context for different values of the spatial covariance factor $\rho$. More precisely, for 11 values of $\rho$ regularly varying from 0 (uncorrelated channels) to 1 (the two channels are identical), 500 bivariate white noises (1024 samples, bidimensional-normal distribution with unitary variance and $\rho$ covariance) are generated. For each signal, the averaged number of oscillation extrema, $\overline{N_{e x t}}$, is computed and fitted in $\log _{2}$ coordinates. Fitting errors and associated slope values are depicted in figure 5. The low fitting error suggests that the spatial covariance doesn't influence the filter bank structure. Regarding the slope, it clearly seems to vary with $\rho$ values. For $\rho=0$, an uncorrelated bivariate white noise is decomposed and the slope value (around -0.48) is close to the one found in the first bivariate simulation. For $\rho=1$, the two signal channels are identical, and the decomposition is "equivalent" to a monovariate uncorrelated decomposition. 
The slope value (around -0.62) is thus close to the one found in the first monovariate simulation.

Application to multichannel sleep recording. The filter bank structure property enjoyed by X-EMD strongly suggests the use of the proposed algorithm in order to analyze the frequential content of real multivariate signals. One example of application dealing with multi-channel sleep recording is proposed here. Therefore, an ambulatory sleep staging system [21] with a reduced number of electrodes (four) is used. It makes possible the record of quadrivariate $(D=4)$ non-invasive electrophysiological observation by means of two temporal (FP1 and FP2) and two frontal (F7 and F8) electrodes. The use of a limited number of electrodes makes impossible the separation of the different electrophysiological sources composing the recorded quadrivariate EEG signal, namely neuronal electrical activity, Slow ElectroOculoGram (SEOG), Rapid ElectroOculogram (REOG) and ElectroMyoGram (EMG). Moreover, these signals are generally corrupted by ElectroCardioGram (ECG) activity.

Extraction of the EMG activity from the quadrivariate observation is a necessary step in the night sleep analysis. The classical procedure to extract this component takes advantage of the specific high-frequency content of the EMG. The EMG component is thus obtained by a simple high-pass filtering (with a $30 \mathrm{~Hz}$ corner frequency), independently on each of the four channels of the original observation [21]. What we propose here is to extract the EMG component by taking advantage of the multivariate filter bank structure of XEMD. It is noteworthy that the challenge here is not to propose a new method in order to extract the EMG signal in sleep recording, but to show how one 

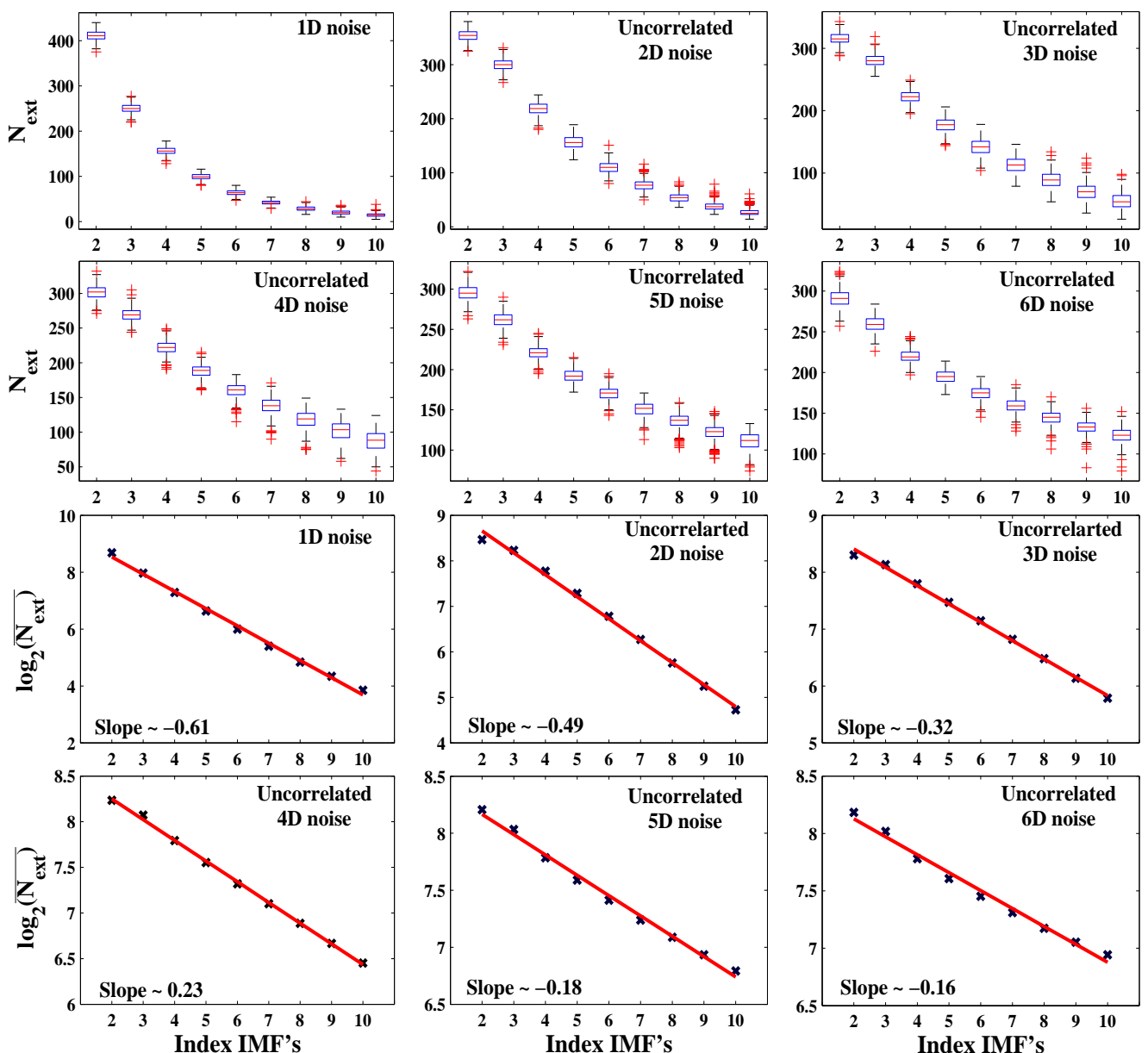

Figure 4: X-EMD filter bank structure: i) top (first and second lines): number of oscillation extrema, $N_{\text {ext }}$, with boxplots as a function of the index of IMFs for uncorrelated white noises of dimensions : $D=1, D=2$ and $D=3, D=4, D=5$ and $D=6$, ii) bottom (third and fourth lines): averaged number of oscillation extrema, $\overline{N_{e x t}}$ (cross) as a function of the index of IMFs in $\log _{2}$ coordinates and the associated linear regression model (continuous line). 

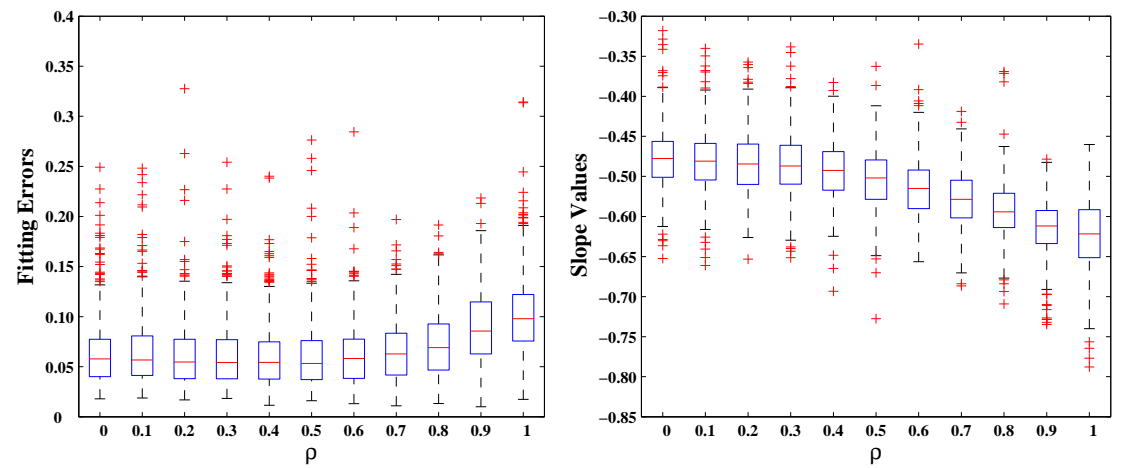

Figure 5: Influence of channel correlation on the filter bank structure: fitting errors vs. $\rho$ values (left), and slope vs. $\rho$ values (right).

could practically take advantage of the X-EMD algorithm. A quadrivariate application of X-EMD to the quadrivariate observation $\mathrm{O}$ is therefore performed on four IMFs in a supervised way. The sum of the first four obtained IMFs $\mathrm{O}_{\mathrm{X}-\mathrm{EMD}}^{\mathrm{HF}}=\left[\mathrm{FP} 1_{\mathrm{X}-\mathrm{EMD}}^{\mathrm{HF}}, \mathrm{FP} 2_{\mathrm{X}-\mathrm{EMD}}^{\mathrm{HF}}, \mathrm{F} 7_{\mathrm{X}-\mathrm{EMD}}^{\mathrm{HF}}, \mathrm{F} 8_{\mathrm{X}-\mathrm{EMD}}^{\mathrm{HF}}\right]^{\top}$ and the associated residue $\mathrm{O}_{\mathrm{X}-\mathrm{EMD}}^{\mathrm{LF}}=\left[\mathrm{FP} 1_{\mathrm{X}-\mathrm{EMD}}^{\mathrm{LF}}, \mathrm{FP} 2_{\mathrm{X}-\mathrm{EMD}}^{\mathrm{LF}}, \mathrm{F} 7_{\mathrm{X}-\mathrm{EMD}}^{\mathrm{LF}}, \mathrm{F} 8_{\mathrm{X}-\mathrm{EMD}}^{\mathrm{HF}}\right]^{\top}$ are then, respectively, compared in figure 6 to the EMG component isolated by the classical high-pass filtering $\mathrm{O}_{\mathrm{FIR}}^{\mathrm{HF}}=\left[\mathrm{FP} 1_{\mathrm{FIR}}^{\mathrm{HF}}, \mathrm{FP} 2_{\mathrm{FIR}}^{\mathrm{HF}}, \mathrm{F} 7_{\mathrm{FIR}}^{\mathrm{HF}}, \mathrm{F} 8_{\mathrm{FIR}}^{\mathrm{HF}}\right]^{\mathrm{T}}$ and the associated residual low-frequency signal $\mathrm{O}_{\mathrm{FIR}}^{\mathrm{LF}}=\left[\mathrm{FP} 1_{\mathrm{FIR}}^{\mathrm{LF}}, \mathrm{FP} 2 \mathrm{\textrm {FIR }}\right.$, $\left.\mathrm{F} 77_{\mathrm{FIR}}^{\mathrm{LF}}, \mathrm{F} 8_{\mathrm{FIR}}^{\mathrm{HF}}\right]^{\top}$.

The channels of the EMG signal estimated by using X-EMD (line 2) and the EMG signals obtained with a highpass filtering (line 4) are, visually, quasi-equivalent. Indeed, the correlation coefficients computed between each channel are comprised between 0.8095 and 0.8320 . This high correlation is also observed on the associated low-frequency residual parts (line 3 and 5). Those last results show that the direct quadrivariate X-EMD method, which 


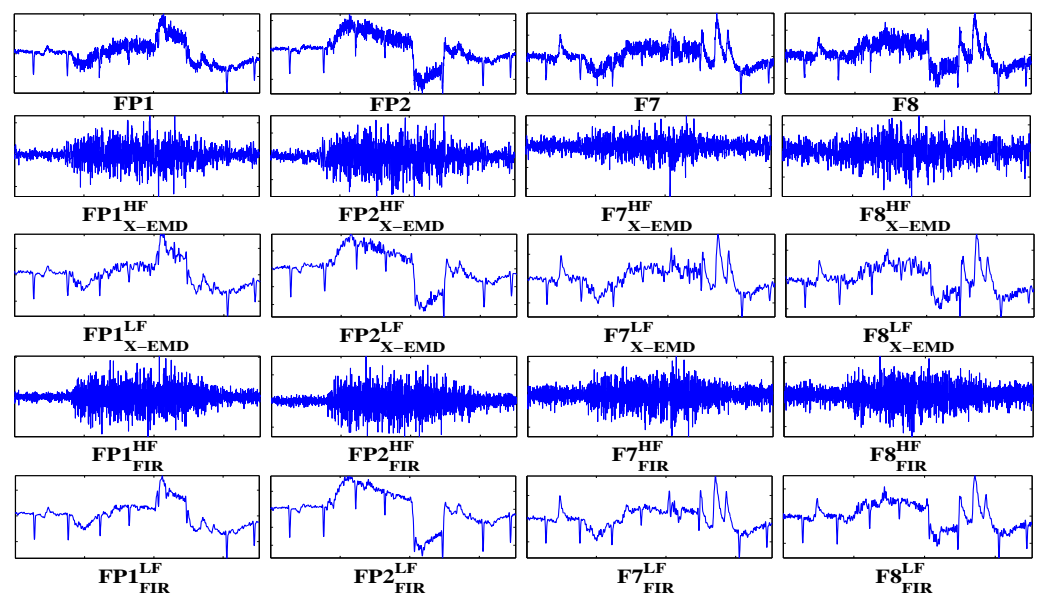

Figure 6: Application of X-EMD to multichannel sleep recording.

takes advantage of the mutual information contained in the four channels, preserves the frequential contents on each channel. Such results may be very interesting in practical contexts but they require a priori regarding the processed signal in order to select the suited IMFs. More particularly, an analysis of the broadband noise present in the signal to process should be performed with X-EMD beforehand. In the biomedical context considered here the broadband noise is essentially due to the neuronal electrical activity. It is noteworthy that if no broadband noise is present in the considered application, it could be artificially added to the data.

\section{Conclusion and perspectives}

A new algorithm called X-EMD based on a redefinition of the local mean operator is proposed. The obtained results show that, under certain assumptions on the processed signal, this alternative definition enables to decompose 
both mono- and multivariate signals without any modification in the core of the algorithm. This last point is the main difference regarding the existing approaches of the literature. The comparative study also suggests that X-EMD seems to offer competitive performance whereas the computational complexity analysis suggests that no loss in terms of numerical performance should be observed in comparison with the existing EMD approaches. Moreover filter bank properties of the classical EMD seem to be preserved and applicable to multivariate signal denoising. A restricted scope of application mainly due to the use of the first derivative in the algorithm remains the most important limitation of our approach. However, as shown in the application of X-EMD in quadrivariate sleep recording, those limitations should be not too much restrictive in practical fields. More simulated and real data decompositions will be performed in future works.

\section{Acknowledgment}

We which acknowledge all the reviewers and the associate editor for their valuable comments. This work is supported by the French Government under an ANR Contract, namely mv-EMD (BLAN07-0314-02).

[1] N. Huang, Z. Shen, S. Long, M. Wu, H. Shih, Q. Zheng, N.-C. Yen, C. Tung, and H. Liu, "The empirical mode decomposition and the hilbert spectrum for nonlinear and non-stationary time series analysis," Proc. R. Soc. London, Ser. A (1998).

[2] L. Hualou, L. Qiu-Hua, and J. Chen, "Application of the empirical mode decomposition to the analysis of esophageal manometric data in gastroe- 
sophageal reflux disease," IEEE Trans. on Bio. Eng., 52(10) (2005) 620623.

[3] M. A. Arafat, J. Sieed and Md. Kamrul Hasan, "Detection of ventricular fibrillation using empirical mode decomposition and Bayes decision theory", Comput. Biol. Med., 39(11) (2009) 1051-1057.

[4] K.T. Coughlin and K.K. Tung, "11-Year solar cycle in the stratosphere extracted by the empirical mode decomposition method," Adv. in Space Res., 34(2) (2004) 323-329.

[5] Y. Huang, F. Schmitt, Z. Lu and Y. Liu, "Analysis of daily river flow fluctuations using Empirical Mode Decomposition and arbitrary order Hilbert spectral analysis," J. of Hydro., 373(1-2) (2009) 103-111.

[6] F. Zhou, M. Xing, X. Bai, G. Sun and Z. Bao, "Narrow-Band Interference Suppression for SAR Based on Complex Empirical Mode Decomposition," IEEE Geosc. and Rem. Sens. Let., 6(3) (2009) 423-427.

[7] N. Chatlani and J. Soraghan, "EMD-based Noise Estimation and Tracking (ENET) with application to speech enhancement," 17th European Signal Processing Conference (EUSIPCO), Glasgow, Scotland, 2009.

[8] N. Bi, Q. Sun, D. Hunag, Z. Yang and J. Huang, "Robust Image Watermarking Based on Multiband Wavelets and Empirical Mode Decomposition," IEEE Trans. on Im. Proc., 16(8) (2007) 1956-1966.

[9] E. Delechelle, J. Lemoine, and O. Niang, "Empirical mode decomposition: an analytical approach for sifting process," IEEE Sig. Proc. Let., 12(11) (2005) 764-767. 
[10] Y. Kopsinis and S. McLaughlin, "Investigation and Performance Enhancement of the Empirical Mode Decomposition Method Based on a Heuristic Search Optimization Approach," IEEE Trans. on Sig. Proc., 56(1) (2008) 1-13.

[11] S.D. Hawley, L.E. Atlas and H.J. Chizeck, "Some Properties of an Empirical Mode Type Signal Decomposition Algorithm," IEEE Sig. Proc. Let., 17(1) (2010) 24-27.

[12] Z. Wu and N.E. Huang, "A study of the characteristics of white noise using the empirical mode decomposition method," Proc. R. Soc. London, 460 (2004) 1597-1611.

[13] P. Flandrin, G. Rilling and P. Goncalves, "Empirical Mode Decomposition as a Filter Bank," IEEE Sig. Proc. Lett., 11(2) (2004) 112-114.

[14] T. Tanaka and D. Mandic, "Complex empirical mode decomposition," IEEE Sig. Proc. Let., 14(2) (2007) 101-104.

[15] M. Bin Altaf, M. Gautama, T. Tanaka, and D. Mandic, "Rotation invariant complex empirical mode decomposition," IEEE International Conference on Acoustics, Speech and Signal Processing (ICASSP07), Honolulu, Hawai, 3 (2007).

[16] G. Rilling, P. Flandrin, P. Goncalves, and J. Lilly, "Bivariate empirical mode decomposition," IEEE Sig. Proc. Let., 14(12) (2007) 1-10.

[17] N. Ur Rehman and D.P. Mandic, "Empirical Mode Decomposition for Trivariate Signals," IEEE Trans. on Sig. Proc., 58(3) (2010) 1059-1068. 
[18] N. Ur Rehman and D.P. Mandic, "Multivariate empirical mode decomposition," Proc. R. Soc. London, Ser. A, 466(2117) (2010) 1291-1302.

[19] J. Fleureau, A. Kachenoura, J.-C. Nunes, L. Albera and L. Senhadji, "3A-EMD: a generalized approach for monovariate and multivariate EMD," in 10th International Conference on Information Sciences, Signal Processing and their Applications (ISSPA 10), pp. 300-303, Kuala Lumpur, Malaysia, 5 (2010).

[20] J. Fleureau, J.-C. Nunes, A. Kachenoura, L. Albera and L. Senhadji, "Turning Tangent Empirical Mode Decomposition: a unified framework for mono- and multivariate signals," IEEE Trans. on Sig. Proc, (under revision).

[21] F. Porée, A. Kachenoura, H. Gauvrit, C. Morvan, G. Carrault and L. Senhadji, "Blind Source Separation for Ambulatory Sleep Recording," IEEE Trans. on Inf. Tech. in Biomed., 10(2) (2006) 293-301. 